\title{
Quantum entanglement in a many-body system exhibiting multiple quantum phase transitions
}

\author{
C. Zander \\ Physics Department, University of Pretoria, Pretoria 0002, South Africa \\ A. Plastino \\ Exact Sciences Fac. - CCT-CONICET, \\ National University La Plata \\ C. C. 727 - 1900 La Plata - Argentina \\ A. R. Plastino \\ Instituto Carlos I de Física Teórica, Universidad de Granada, Granada, Spain, EU \\ National University La Plata, UNLP-CREG-CONICET \\ C. C. 727 - 1900 La Plata - Argentina and \\ Physics Department, University of Pretoria, Pretoria 0002, South Africa
}

(Received on 15 December, 2008)

\begin{abstract}
We investigate the quantum entanglement-related features of the many-body model of Plastino and Moszkowski [N. Cimento 47 (1978) 470]. This is an exactly solvable N-body, SU2 two-level model exhibiting several quantum phase transitions. We show that these transitions happen to be also entanglement-transitions associated with different many-body Dicke states. The main properties of the model considered here make it particularly well suited to study, by recourse to exact analytical computations, some connections between quantum phase transitions and quantum entanglement-theory.
\end{abstract}

Keywords: Quantum phase transitions, Many body systems

\section{INTRODUCTION}

A quantum phase transition (QPT) is a phase transition between different quantum phases (phases of matter at zero temperature). Contrary to classical phase transitions, quantum phase transitions can only be accessed by varying a physical parameter - such as magnetic field or pressure - at absolute zero temperature. The transition describes a structural change in the ground state of a many-body system. Such quantum phase transitions can be first-order phase transition or continuous. To understand quantum phase transitions, it is useful to contrast them to classical phase transitions (CPT) (also called thermal phase transitions). A CPT describes a cusp in the thermodynamic properties of a system. It signals a reorganization of the particles. A typical example is the freezing transition of water describing the transition between liquid and ice. The classical phase transitions are driven by a competition between the energy of a system and the entropy of its thermal fluctuations. A classical system does not have entropy at zero temperature and therefore no phase transition can occur.

In contrast, even at zero temperature, a quantum-mechanical system can still support phase transitions. As a relevant physical quantity is varied (represented by a parameter in the system's Hamiltonian) it is possible to induce a phase transition into a different phase of matter. A paradigmatic example of a quantum phase transition is the well-studied superconductor/insulator transition in disordered thin films which separates two quantum phases having different symmetries. Quantum magnets provide another example of QPT. Thus, in infinite as well as in finite systems a type of phase transition, often referred to as a quantum phase transition, may occur at $\mathrm{T}=0$. Such quantum phase transitions generally signal a change in the correlations present in the ground state of the system. For a system described by a Hamiltonian, $H(\xi)=H_{0}+\xi H_{1}$, which varies as a function of the coupling constant $\xi$, the presence of a QPT can easily be understood in the following manner: level crossing may come about and the ground state energy is no longer analytic nor monotonic. Although there are other valid mathematical reasons that lead to the loss of analyticity, the above simple explanation will suffice for our purposes and provides a simple means for defining a QPT.

\section{FORMALISM TO BE EMPLOYED}

We briefly describe below the formalism that we are going to use in order to detect the quantum phase transitions exhibited by the $N$-body models studied here. For more details see [1]. It is clear that the quantum entanglement properties associated with these quantum transitions (which constitute the main focus of the present study) do not depend on the particular method employed to detect them. However, we think that the method explored in [1] is particularly appropriate for our present goals, because it may stimulate new lines of enquiry related to the temperature dependence of the entanglement features exhibited by the Plastino-Moszkowski and related models. Consider a system whose dynamics is described (at $T=0$ ) by the following Hamiltonian operator

$$
\hat{H}=\hat{H}_{0}+\xi \hat{H}_{1} .
$$

At finite temperatures, the Maximum Entropy Principle of Jaynes [2] can be used to determine the appropriate statistical operator, $\hat{\rho}$ in the following manner. Maximizing the entropy, $S(\hat{\rho})=\operatorname{Tr}[\hat{\rho} \log \hat{\rho}]$,

$$
\delta_{\rho} S(\hat{\rho})=0,
$$

subject to the constraints

$$
<\hat{H}>=\operatorname{Tr}[\hat{\rho} \hat{H}]=\mathcal{E},
$$


and

$$
\operatorname{Tr}[\hat{\rho}]=1
$$

yields

$$
\hat{\rho}=\frac{\exp (-\beta \hat{H})}{Z}
$$

where

$$
Z=\operatorname{Tr}\left[e^{-\beta \hat{H}}\right]
$$

Generally, in statistical mechanics the coupling constant $\xi$ is taken to be a constant and equation (2) is used to determine the Lagrange multiplier $\beta$. Our basic idea to approach QPTs is to allow for $\xi$ to vary. Thus, $\xi$ no longer being a constant, one becomes entitled to look for a functional relation between $\beta$ and $\xi$, using equation (3) and common thermo-statistical ideas.

For instance, the specific heat is given by an $\hat{H}$-fluctuation, namely,

$$
\begin{aligned}
C_{\beta} & =-\beta^{2}\left[\left\langle\hat{H}^{2}\right\rangle-\langle\hat{H}\rangle^{2}\right] \\
& =-\beta^{2}\left(\frac{\partial<\hat{H}>}{\partial \beta}\right)_{\xi}
\end{aligned}
$$

The guiding idea now is to make an analogy with (Nerst's) thermodynamics' third-law in order to establish a criterion for detecting the critical values $\xi_{C}$ of the coupling constant leading to a structural change in the system's ground state. As discussed in detail in reference [1], in the Plastino-Moszkowski model these critical values can be determined by considering the limit $T \rightarrow 0$ (or, equivalently $\beta \rightarrow \infty$ ) in the relations

$$
\left(\frac{\partial}{\partial \beta}<\hat{H}>\right)_{\xi}=0,
$$

or

$$
\left(\frac{\partial}{\partial \xi}<\hat{H}>\right)_{\beta}=0 .
$$

When applying equation (9) one has to note that

$$
\left(\frac{\partial<\hat{H}>}{\partial \xi}\right)_{T=0}=\frac{\partial E_{g s}}{\partial \xi} .
$$

since only the ground state is populated at that temperature. If, indeed, as has already been pointed out, a QPT occurs at a level crossing then two possibilities exist:

1. A discontinuous derivative

$$
G(\xi)=\left(\frac{\partial E_{g s}}{\partial \xi}\right)_{\beta=\infty}(\xi),
$$

if $\frac{\partial E_{g s}}{\partial \xi}$ does not change sign when passing through $\xi_{c}$.

2. A null derivative at $\xi_{c}$.
For finite systems at finite temperatures $(T \neq 0), C_{\beta}$ is analytic and structures in $\frac{\partial<H>}{\partial \beta}$ should be indicative of the remnant of a phase transition. The relation $\left(\frac{\partial}{\partial \beta}<\hat{H}>\right)_{\xi}=0$ allows one to correctly determine the position of the QPT. Alternatively $\frac{\partial E_{g s}}{\partial \xi}$ can be used in the manner outlined above to determine the position of a QPT. These two procedures are equivalent, as demonstrated in [1]. In the next section we are going to analyze the quantum entanglement changes associated with the quantum phase transitions exhibited by a genuine nuclear physics' many-body model.

\section{THE PLASTINO-MOSZKOWSKI MODEL}

This is an exactly solvable N-body, SU2 two-level model [3]. Each level can accommodate an even number $N$ of particles, i.e., is $N$-fold degenerate. There are two levels separated by an energy gap, say $\varepsilon=1$, and occupied by $N$ particles. In the model the angular momentum-like operators $J^{2}, J_{x}, J_{y}, J_{z}$, with $J(J+1)=N(N+2) / 4$ are used (see details below). The Hamiltonian is given by [3]

$$
H=J_{z}-\xi\left[J^{2}-J_{z}^{2}-N / 2\right]
$$

States belonging to the lowest lying multiplet, i.e., that with $J=N / 2$, are usually referred to as Dicke-states $|J, M\rangle(M$ standing for the $J_{z}$-eigenvalues) [4]. We use, in building up our many-body Hamiltonian, the second quantization form

$$
J_{z}=\frac{1}{2} \sum_{i=1}^{N} \sum_{\sigma=1}^{2} a_{i, \sigma}^{\dagger} a_{i, \sigma},
$$

with corresponding expressions for $J_{x}, J_{y}$. This is a simple yet nontrivial case of the Lipkin model [5]. Note that we deal here with a bonafide many-body system, since the number of states grows with $N$ as $2^{N}$. At the beginning we will discuss only the model in the zero-temperature regime. The operators appearing in the model Hamiltonian form a commuting set of observables and are thus simultaneously diagonalizable.

\subsection{QPTs in the PM-model}

The ground state of the unperturbed system $(\xi=0$ and at $T=0)$ is $|J, M\rangle=\left|\frac{N}{2},-\frac{N}{2}\right\rangle$ with the eigen-energy $E_{0}=-\frac{1}{2} N$. When the interaction is turned on $(\xi \neq 0)$ and gradually becomes stronger, the ground state energy will in general be different from the unperturbed system for some critical value of $\xi$ that we will call $\xi_{c}$. This sudden change of the ground state energy signifies a quantum phase transition. It should be noted that for a given value of $N$, there could be more than one critical point, since there are $2 J+1$ possible values for $M$. If we denote by $n$ the number of "holes" in the lowest of the two $N$-degenerate levels, then the critical value $\xi_{c}$ of the $n$th transition point can be found from equation (14) below, provided that $\xi_{c}>0$ and $\xi_{c} \neq \infty[3]$

$$
\xi_{c, n}=\frac{1}{N-(2 n-1)} .
$$




\section{DICKE-STATES' 2-QUBITS ENTANGLEMENT}

Remember that for these states $J=N / 2$ and $M=-J, \ldots, J$ [6]. In the computational basis

$$
|00\rangle,|01\rangle,|10\rangle,|11\rangle,
$$

one has a reduced two-body matrix [6]

$$
\rho_{12}=\left(\begin{array}{cccc}
v_{+} & 0 & 0 & 0 \\
0 & y & y & 0 \\
0 & y & y & 0 \\
0 & 0 & 0 & v_{-}
\end{array}\right),
$$

with [6]

$$
\begin{aligned}
v_{ \pm} & =\frac{(N \pm 2 M)(N-2 \pm 2 M)}{4 N(N-1)} \\
y & =\frac{N^{2}-4 M^{2}}{4 N(N-1)}
\end{aligned}
$$

and concurrence,

$$
C\left[\rho_{12}\right]=2 \operatorname{Max}\left(0, y-\sqrt{\left(v_{-} v_{+}\right)}\right),
$$

where, as stated, $C\left[\rho_{12}\right]$ stands for the all-important quantity that measures the entanglement-degree, namely, the concurrence. We immediately realize that at the extremal multiplet values $M= \pm J$ one has $y=0, C\left[\rho_{12}\right]=0$, so that these are two separable states, and, further,

$$
\begin{array}{ll}
v_{+}=1 ; v_{-}=0 & M=N / 2 \\
v_{+}=0 ; v_{-}=1 & M=-N / 2 .
\end{array}
$$

Instead, for $M=0$ we get a "minimal-finite" entanglement amount, i.e., that particular many-body state with this $M$-value is the least entangled non-separable Dicke state, for which

$$
\begin{aligned}
y & =\frac{N}{4(N-1)} \\
v_{ \pm} & =\frac{N-2}{4(N-1)},
\end{aligned}
$$

leading to

$$
\begin{aligned}
C\left[\rho_{12}\right] & =\frac{1}{N-1} \\
\lim _{N \rightarrow \infty} C\left[\rho_{12}\right] & =0 .
\end{aligned}
$$

Notice that $C\left[\rho_{12}\right] \leq 1$ for $N \geq 2$. For given $N$, the concurrence is always maximal for $M= \pm \frac{N}{2} \mp 1$, which is a so-called W-state [7, 8]. In terms of the number of holes $n$ in the lowest lying of our two levels, the $\mathrm{W}$-states are those with $(n=1$ or $n=\frac{J}{2}-1$. The pertinent concurrence becomes $C_{W}=\frac{2}{N}$. These are known to be maximally entangled states in general. For an arbitrary Dicke-state the concurrence reads

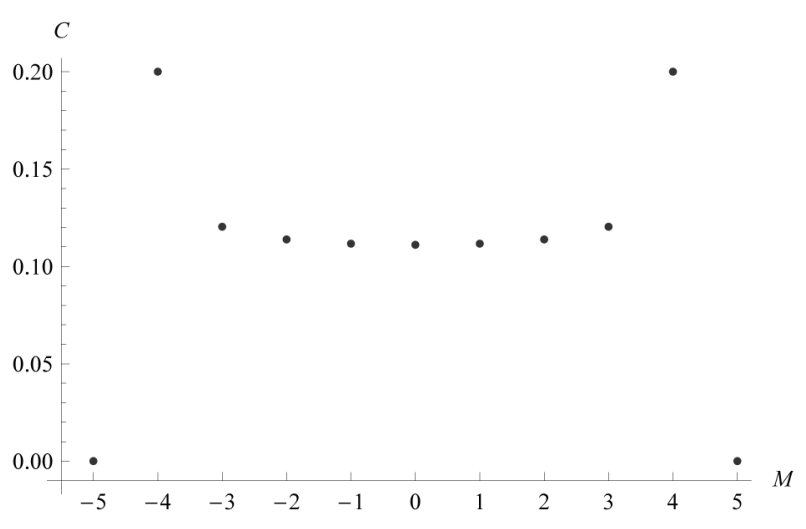

FIG. 1: Concurrence of the ground states of the PlastinoMoszkowski model with $N=10$ particles as a function of the quantum number $M$. All depicted quantities are dimensionless.

$$
C\left[\rho_{12}\right]=\frac{N^{2}-4 M^{2}-\sqrt{\left(N^{2}-4 M^{2}\right)\left[(N-2)^{2}-4 M^{2}\right]}}{2 N(N-1)},
$$

leading to the limit value

$$
\lim _{N \rightarrow \infty} C\left[\rho_{12}\right]=0 .
$$

\subsection{QPT and entanglement}

The ground state of the unperturbed system $(\xi=0$ and at $T=0$ ), i.e., $|J, M\rangle=\left|\frac{N}{2},-\frac{N}{2}\right\rangle$ is a separable state. When the interaction is turned on $(\xi \neq 0)$ and gradually becomes stronger, the ground state energy will in general be different from the unperturbed system for critical values $\xi_{c}$. Such sudden change of the ground state energy signifies both a quantum phase transition and an entanglement transition. As, for a given value of $N$, there exists more than one critical point (indeed $N-1$ ones) we have a matching set of sudden entanglement changes. The first and the last of such transitions are, respectively, from a separable to a maximally entangled state and vice versa. Thus, at the $n$th transition $\xi_{c}$, where the number of holes $n$ grows by one, and $\xi_{c, n}=\frac{1}{N-(2 n-1)}$, the ground state of the many-body system becomes a new kind of entangled state.

\subsection{Thermodynamic limit}

Notice that the very presence of other particles, even without interaction, diminishes the concurrence of $\rho_{12}$. What happens in the thermodynamic limit $N \rightarrow \infty$ ?

In answering, we can, without loss of generality, limit our considerations to the W-states, whose concurrence steadily diminishes with $N$. Obviously, $C\left[\rho_{12}\right]$ vanishes then in the thermodynamic limit, which is, in a way, a classical one. This 
is consistent with other instances of quantum systems adopting classical-like properties in the limit cases corresponding to a large number of particles or a large number of degrees of freedom $[8,9]$. An interesting example of this kind of behavior concerns a typical feature of the dynamics of multipartite quantum systems: for some quantum states entanglement enhances the "speed" of quantum evolution, as measured by the time $\tau$ required to reach a state orthogonal to the initial one [8]. This evolution time $\tau$ has a lower bound $\tau_{\min }$ determined by the energy resources of the quantum state under consideration. When one has a system of $N$ independent qubits a certain amount of entanglement is always needed in order to saturate the aforementioned bound on the evolution time. However, when the number of qubits goes to infinity the entanglement required to reach this "quantum speed limit" goes to zero [8], which may be interpreted as a classical-like feature exhibited by the $N \rightarrow \infty$ limit.

\section{CONCLUSION}

We have in this note obtained some significant new results: 1) The Plastino-Moszkowski model [3] exhibits a rich variety of entangled states. The transition between them takes place precisely at those critical values of the coupling constant for which a quantum phase transition occurs.

2) The above makes the Plastino-Moskowski system a useful solvable model to study the link between entanglement and QPTs in many-body systems.

3) In the thermodynamic limit, which in a sense may be regarded as the classical limit, one analytically ascertains that the entanglement of the many-body system vanishes, as it should.

4) The PM model was advanced to exhibit inadequacies of the Hartree-Fock approach [3], which is unable to detect the QPT's. This fact is illuminated here on the basis of the QPTentanglement link that we have investigated in the present contribution. The Hartree-Fock state is represented by one single Slater determinant and is, consequently, always a separable state. In fact, in modern quantum mechanical terminology, the Hartree-Fock approach can be described as a "zero entanglement" approximation.

\section{Acknowledgments}

This work was partially supported by the Projects FQM2445 and FQM-207 of the Junta de Andalucia (Spain, EU). The financial assistance of the National Research Foundation (NRF; South African Agency) towards this research is hereby acknowledged. Opinions expressed and conclusions arrived at, are those of the authors and are not necessarily to be attributed to NRF.
[1] M. Kruse, H. Miller, A. Plastino, A. R. Plastino, arXiv 0805.4271v1[nucl-th].

[2] E. T. Jaynes, Phys. Rev. 106 (1957) 620; 108 (1957) 171; Papers on probability, statistics and statistical physics, edited by R. D. Rosenkrantz (Reidel, Dordrecht, Boston, 1987); A. Katz, Principles of Statistical Mechanics (Freeman and Co., San Francisco, 1967).

[3] A. Plastino, S. A. Moszkowski, N. Cimento 47 (1978) 470.

[4] R. H. Dicke, Phys. Rev. 93 (1954) 99.

[5] D. Agassi, H. J. Lipkin, N. Meshkov, Nucl. Phys. 86 (1966) 321;
H. J. Lipkin, N. Meshkov, A. J. Glick, Nucl. Phys. 62 (1965) 188; A. Faessler, A. Plastino, Zeit. Phys. 239 (1970) 73.

[6] X. Wang, K. Moellmer, Eur. Phys. J. D 18 (2002) 385; H. T. Cui, Phys. Rev. A 77 (2008) 052105.

[7] A. Borras, A.R. Plastino, J. Batle, C. Zander, M. Casas and A. Plastino, J. Phys. A: Math. and Theor. 40 (2007) 13407.

[8] C. Zander, A.R. Plastino, A. Plastino, and M. Casas, J. Phys. A: Math. and Theor. 40 (2007) 2861.

[9] L.G. Yaffe, Physics Today 36 August (1983) 50. 